\title{
Principaux résultats de l'enquête effectuée en 2006 auprès des médecins-assistants
}

\author{
Pascale Orlow, \\ Michael Siegrist ${ }^{a}$, \\ Max Giger ${ }^{b}$ \\ a Centre de recherche sociale de \\ l'Université de Zurich \\ b Département de la formation \\ médicale de la FMH
}

Correspondance:

Michael Siegrist, Dr ès lettres, p.-d. Centre de recherche sociale de l'Université de Zurich Binzmühlestrasse 14 / Box 15 CH-8050 Zurich

\begin{abstract}
Résumé
En 2006, les médecins-assistants briguant un titre fédéral de formation postgraduée ont été invités pour la dixième fois à évaluer leur formation postgraduée. Le taux de réponses s'est élevé à 65\%. Depuis 2003, le nombre de places de formation postgraduée clinique a augmenté de $8 \%$ pour passer à 8626 . Durant la même période, le temps de travail moyen hebdomadaire a diminué de 2,5 heures environ pour passer à 54,2 heures. La durée moyenne de fréquentation des cours théoriques est restée stable et a même augmenté dans certains domaines. De 2003 à 2006, la part des médecins-assistants disposant d'un diplôme suisse de fin d'études a reculé de $7 \%$ pour passer à $60,8 \%$. La formation postgraduée est jugée bonne dans l'ensemble des établissements, à l'exception de quelquesuns, et - à l'instar des enquêtes précédentes comme très bonne dans les petits établissements. L'étendue des compétences transmises diffère selon leur importance dans la discipline concernée. 92\% des médecins-assistants choisissent leur titre de spécialiste dans les trois ans qui suivent le diplôme de fin d'études. Sur une période de cinq ans après le diplôme, on constate une augmentation des titres brigués en psychiatrie et médecine interne, alors que le nombre des titres visés en chirurgie diminue et celui des titres visés en médecine générale reste stable.
\end{abstract}

La dixième enquête visant à évaluer la formation postgraduée par les médecins-assistants a été effectuée durant les mois de juillet à novembre 2006. Pour cette enquête, nous avons utilisé le questionnaire de l'année 2005 en y apportant quelques petites modifications. A la différence des premières enquêtes, des questions explicites ont été posées sur les contenus enseignés. C'est ainsi que le sondage a également porté sur la contribution de l'établissement de formation postgraduée au développement des différentes compétences, aptitudes et connaissances du point de vue des médecins-assistants.
En décembre 2006, les responsables des établissements de formation postgraduée ont reçu un compte rendu détaillé avec les résultats de leur établissement et les valeurs comparatives des autres établissements de la même discipline. 187 établissements de formation postgraduée ne l'ont pas reçu car ils n'avaient pas renvoyé de questionnaires (utilisables). Cette année également, une vue d'ensemble des comptes rendus est publiée sur l'internet.

Le présent article présente quelques résultats importants sous forme résumée. Pour certaines questions choisies, nous comparons les résultats actuels avec ceux d'enquêtes précédentes.

\section{Méthode}

\section{Ensemble des personnes sondées}

Le questionnaire a été envoyé à 1175 responsables d'établissements de formation postgraduée. Ces responsables ont été chargés de le distribuer à 8626 médecins-assistants (5799 en allemand et 2827 en français). Ceux-ci pouvaient renvoyer les questionnaires remplis directement à l'Institut de recherche sociale de l'Université de Zurich, responsable de l'enquête. Le taux de réponses s'est élevé à $65 \%(n=5641)$. Cette valeur est comparable au taux de réponses des enquêtes précédentes [1-3]. 49\% des questionnaires ont été remplis par des assistants femmes et 51\% par des hommes.

\section{Questionnaire}

Le questionnaire contenait 101 items. Les sondés devaient avant tout prendre position sur un certain nombre d'affirmations. Pour la plupart de celles-ci, six réponses étaient possibles, allant de 1 «ce n'est pas du tout exact» à 6 «c'est parfaitement exact». Concernant la contribution des établissements à la transmission des compétences professionnelles, les médecins-assistants disposaient d'une liste répertoriant des compétences concrètes et pouvaient, pour chacune d'entre elles, évaluer ladite contribution de «très petite» à «très grande», ou cocher l'option «non applicable». Le principe suivant s'applique de 
manière générale aux dimensions calculées (valeurs moyennes des items): plus la valeur de la dimension est élevée, meilleur est le résultat obtenu dans la dimension concernée. Le module constitué de questions supplémentaires et changeant chaque année contenait en 2006 des questions sur les raisons à la base du choix de la spécialisation.

\section{Résultats}

\section{Transmission des compétences} professionnelles

Les médecins-assistants pouvaient indiquer quelle était la contribution fournie par l'établissement de formation postgraduée au développement de diverses compétences, aptitudes et connaissances. Il ressort du tableau 1 que ces compétences sont pondérées différemment selon la discipline dans laquelle les assistants sont engagés. Le tableau montre les six disciplines comptant le plus de médecins-assistants. La chirurgie est la discipline la plus mal évaluée pour la transmission de presque toutes les compétences concernées. Dans les établissements de formation postgraduée en chirurgie et chirurgie orthopédique, la transmission d'une pharmacothérapie efficace et sûre se voit conférer trop peu d'importance. L'aptitude à évaluer la capacité de travail du patient est transmise de manière insuffisante en médecine interne, chirurgie et gynécologie/obstétrique.

La discipline en soi n'est pas le seul facteur à influencer l'évaluation des compétences acquises. Dans certaines disciplines, la grandeur (nombre de médecins-assistants) de l'établissement de formation postgraduée joue également un rôle. C'est particulièrement le cas en médecine interne, anesthésiologie et psychiatrie. Pour la formation postgraduée en médecine interne, le tableau 2 indique les différences dans la transmission de compétences d'après la taille de l'établissement. Les médecins-assistants ont estimé que dans les grands établissements, on accorde une importance légèrement moindre aux questions «anamnèse», «évaluation de la capacité de travail du patient», «information claire et compréhensible du patient sur son état de santé» et «principes éthiques de la médecine» que dans les petits établissements. Mais il existe aussi des domaines, comme la «collaboration avec d'autres disciplines médicales», dans lesquels les grands établissements obtiennent une meilleure note que les petits. La grandeur de l'établissement n'exerce aucune influence sur la transmission des «connaissances professionnelles spécifiques à la discipline».
Influence du nombre de médecins-assistants d'un établissement sur l'évaluation globale

L'évaluation globale a été mesurée avec trois items (exemple: «Je suis dans l'ensemble satisfait(e) de ma condition de travail actuelle.»). L'échelle présente une très bonne consistance interne (coefficient alpha $=.94$ ). La taille de l'établissement de formation postgraduée joue un rôle aussi bien dans la transmission des compétences que dans l'évaluation globale de celui-ci. Les valeurs moyennes pour l'évaluation globale, réparties selon la grandeur de l'établissement (nombre de postes d'assistants) et la discipline, figurent dans le tableau 3. Des différences peuvent apparaître, aussi bien entre les disciplines qu'entre établissements petits et grands. De manière générale, les établissements comptant un petit nombre de médecins-assistants obtiennent un meilleur résultat. La chirurgie obtient des valeurs plus basses que les autres disciplines, indépendamment de la grandeur des établissements.

\section{Influence du facteur année de formation spécifique ou année de formation à option sur l'appréciation globale des établissements de formation postgraduée}

Parmi les médecins-assistants qui ont rempli le questionnaire, 78,4\% ( $n=4343)$ accomplissaient une année de formation spécifique et 14,5\% ( $\mathrm{n}=802)$ une année à option. $7,1 \%(\mathrm{n}=395)$ ne s'étaient pas encore décidés pour une discipline.

Les valeurs moyennes de l'évaluation globale, réparties d'après l'année de formation spécifique ou l'année à option des médecins-assistants, figurent dans le tableau 4 pour les sept disciplines comptant le plus d'assistants. En comparaison avec les résultats précédents [3], aucune différence n'a pu être constatée cette année dans l'évaluation globale entre les médecins-assistants accomplissant une formation spécifique et ceux qui accomplissaient une année à option. Répartis par discipline, les premiers n'ont, globalement, pas mieux placé leur établissement de formation que les seconds ou que les médecinsassistants encore indécis. Cette observation est valable pour toutes les disciplines.

Concernant la transmission des compétences professionnelles ainsi que la culture de formation, de gestion et d'entreprise (exceptions pour la psychiatrie), les médecins-assistants en année de formation spécifique ont évalué leur établissement de manière semblable à leurs autres collègues de travail. 
Tableau 1

Valeurs moyennes des réponses concernant la contribution des établissements de formation postgraduée au développement des diverses compétences, aptitudes et connaissances, pour les six disciplines comptant le plus de médecins-assistants.

\begin{tabular}{|c|c|c|c|c|c|c|}
\hline & $\begin{array}{l}\text { Médecine interne } \\
(n=1285)\end{array}$ & $\begin{array}{l}\text { Chirurgie } \\
(n=661)\end{array}$ & $\begin{array}{l}\text { Gynécologie } \\
(n=314)\end{array}$ & $\begin{array}{l}\text { Pédiatrie } \\
(n=268)\end{array}$ & $\begin{array}{l}\text { Psychiatrie } \\
(n=681)\end{array}$ & $\begin{array}{l}\text { Chirurgie ortho- } \\
\text { pédique }(n=198)\end{array}$ \\
\hline Anamnèse & 4.81 & 4.34 & 4.86 & 4.82 & 4.77 & 4.50 \\
\hline Techniques d'examen clinique & 4.45 & 4.29 & 4.73 & 4.48 & 4.25 & 4.74 \\
\hline Conduite d'entretien & 4.53 & 3.96 & 4.65 & 4.33 & 4.77 & 4.18 \\
\hline $\begin{array}{l}\text { Connaissances professionnelles spécifiques } \\
\text { à la discipline }\end{array}$ & 5.07 & 4.77 & 5.01 & 5.01 & 4.94 & 5.23 \\
\hline Application pratique du savoir théorique & 4.89 & 4.54 & 4.78 & 4.80 & 4.91 & 4.72 \\
\hline Raisonnement clinique & 5.07 & 4.56 & 4.76 & 4.89 & 4.84 & 4.81 \\
\hline Pose d'indication pour des examens spéciaux & 4.76 & 4.50 & 4.61 & 4.58 & 4.27 & 4.83 \\
\hline Pose d'indication pour des traitements & 4.95 & 4.64 & 4.82 & 4.84 & 4.75 & 5.08 \\
\hline Accompagnement de mourants & 4.31 & 3.15 & 3.21 & 3.08 & 3.11 & 2.75 \\
\hline $\begin{array}{l}\text { Analyse utilité-risque dans le cadre } \\
\text { des diagnostics et des traitements }\end{array}$ & 4.52 & 4.08 & 4.24 & 4.11 & 4.10 & 4.41 \\
\hline Evaluation de la capacité de travail du patient & 3.47 & 3.90 & 3.93 & 3.04 & 4.45 & 4.45 \\
\hline $\begin{array}{l}\text { Maîtrise des techniques d'intervention et } \\
\text { d'examen clinique (p.ex. opérations, examens } \\
\text { cliniques, examens spéciaux, entretiens) }\end{array}$ & 4.30 & 4.47 & 4.77 & 4.28 & 4.48 & 4.76 \\
\hline Interprétation des résultats d'examens & 4.83 & 4.50 & 4.84 & 4.77 & 4.39 & 4.88 \\
\hline $\begin{array}{l}\text { Utilisation correcte de médicaments } \\
\text { (pharmacothérapie efficace et sûre) }\end{array}$ & 4.78 & 3.90 & 4.39 & 4.69 & 4.88 & 3.86 \\
\hline $\begin{array}{l}\text { Utilisation économique des ressources dans } \\
\text { le cadre des diagnostics et des traitements }\end{array}$ & 4.33 & 4.05 & 4.22 & 4.12 & 4.36 & 4.12 \\
\hline $\begin{array}{l}\text { Application de nouvelles connaissances } \\
\text { scientifiques }\end{array}$ & 4.66 & 3.98 & 4.55 & 4.38 & 4.39 & 4.68 \\
\hline Analyse des erreurs & 4.28 & 4.19 & 4.37 & 4.51 & 4.16 & 4.47 \\
\hline $\begin{array}{l}\text { Information claire et compréhensible } \\
\text { du patient sur son état de santé }\end{array}$ & 4.93 & 4.59 & 4.97 & 4.99 & 4.93 & 4.73 \\
\hline $\begin{array}{l}\text { Information du patient sur les risques } \\
\text { et l'utilité d'une intervention }\end{array}$ & 4.65 & 4.66 & 5.14 & 4.62 & 4.67 & 4.96 \\
\hline Etablissement de rapports médicaux & 4.92 & 4.69 & 4.88 & 4.73 & 4.92 & 4.63 \\
\hline Prise de responsabilités & 4.90 & 4.68 & 4.71 & 4.45 & 5.16 & 4.73 \\
\hline $\begin{array}{l}\text { Collaboration avec d'autres disciplines } \\
\text { médicales }\end{array}$ & 4.84 & 4.65 & 4.59 & 4.56 & 4.60 & 4.71 \\
\hline Collaboration avec le personnel infirmier & 5.03 & 4.86 & 5.05 & 5.19 & 5.16 & 4.76 \\
\hline $\begin{array}{l}\text { Collaboration avec des services } \\
\text { paramédicaux (p.ex. physiothérapie, } \\
\text { ergothérapie, services sociaux) }\end{array}$ & 4.79 & 4.65 & 4.37 & 4.52 & 5.11 & 4.99 \\
\hline $\begin{array}{l}\text { Collaboration avec les médecins / } \\
\text { médecins de premier recours } \\
\text { chargés du suivi }\end{array}$ & 4.65 & 4.39 & 4.39 & 4.51 & 4.89 & 4.37 \\
\hline Evaluation réaliste de ses propres capacités & 4.57 & 4.37 & 4.68 & 4.48 & 4.67 & 4.63 \\
\hline Activité indépendante & 4.97 & 4.79 & 4.97 & 4.63 & 5.28 & 4.87 \\
\hline Principes éthiques de la médecine & 4.56 & 4.04 & 4.65 & 4.41 & 4.80 & 4.29 \\
\hline
\end{tabular}

Remarque: les valeurs allaient de 1 «très petite» (très petite contribution) à 6 «très grande». Seules ont été prises en compte les réponses de médecinsassistants exerçant leur activité dans l'établissement de formation postgraduée depuis deux mois ou plus. Pour les aspects ne concernant pas la discipline exercée, la possibilité de réponse était «non applicable». Ces valeurs-là n'ont pas été prises en compte dans le calcul des valeurs moyennes. 
Tableau 2

Etablissements de formation en médecine interne: valeurs moyennes des réponses concernant la contribution des établissements de formation postgraduée au développement des diverses compétences, aptitudes et connaissances (valeurs réparties en fonction de la taille de l'établissement.

$\mathrm{MA}=$ médecin-assistant).

\begin{tabular}{|c|c|c|c|c|}
\hline & \multicolumn{4}{|l|}{ Médecine interne } \\
\hline & 1 à $3 M A(n=69)$ & 4 à $10 \mathrm{MA}(n=347)$ & 11 MA et plus $(n=869)$ & Total $(n=1285)$ \\
\hline & M & M & M & M \\
\hline Anamnèse & 5.06 & 5.03 & 4.70 & 4.81 \\
\hline Techniques d'examen clinique & 4.72 & 4.69 & 4.33 & 4.45 \\
\hline Conduite d'entretien & 4.93 & 4.70 & 4.43 & 4.53 \\
\hline Connaissances professionnelles spécifiques à la discipline & 5.03 & 5.05 & 5.08 & 5.07 \\
\hline Application pratique du savoir théorique & 4.81 & 5.03 & 4.84 & 4.89 \\
\hline Raisonnement clinique & 4.91 & 5.12 & 5.07 & 5.07 \\
\hline Pose d'indication pour des examens spéciaux & 4.69 & 4.76 & 4.76 & 4.76 \\
\hline Pose d'indication pour des traitements & 4.94 & 4.99 & 4.94 & 4.95 \\
\hline Accompagnement de mourants & 4.48 & 4.62 & 4.18 & 4.31 \\
\hline $\begin{array}{l}\text { Analyse utilité-risque dans le cadre des diagnostics } \\
\text { et des traitements }\end{array}$ & 4.43 & 4.69 & 4.46 & 4.52 \\
\hline Evaluation de la capacité de travail du patient & 4.39 & 3.64 & 3.33 & 3.47 \\
\hline $\begin{array}{l}\text { Maîtrise des techniques d'intervention et } \\
\text { d'examen clinique (p.ex. opérations, examens cliniques, } \\
\text { examens spéciaux, entretiens) }\end{array}$ & 4.17 & 4.46 & 4.25 & 4.30 \\
\hline Interprétation des résultats d'examens & 4.77 & 4.95 & 4.79 & 4.83 \\
\hline $\begin{array}{l}\text { Utilisation correcte de médicaments } \\
\text { (pharmacothérapie efficace et sûre) }\end{array}$ & 4.98 & 4.88 & 4.73 & 4.78 \\
\hline $\begin{array}{l}\text { Utilisation économique des ressources dans le cadre } \\
\text { des diagnostics et des traitements }\end{array}$ & 4.76 & 4.79 & 4.11 & 4.33 \\
\hline Application de nouvelles connaissances scientifiques & 4.63 & 4.54 & 4.71 & 4.66 \\
\hline Analyse des erreurs & 4.21 & 4.42 & 4.24 & 4.28 \\
\hline $\begin{array}{l}\text { Information claire et compréhensible du patient } \\
\text { sur son état de santé }\end{array}$ & 5.36 & 5.01 & 4.86 & 4.93 \\
\hline $\begin{array}{l}\text { Information du patient sur les risques et l'utilité } \\
\text { d'une intervention }\end{array}$ & 4.79 & 4.64 & 4.64 & 4.65 \\
\hline Etablissement de rapports médicaux & 5.08 & 5.11 & 4.83 & 4.92 \\
\hline Prise de responsabilités & 4.91 & 5.08 & 4.82 & 4.90 \\
\hline Collaboration avec d'autres disciplines médicales & 4.53 & 4.78 & 4.89 & 4.84 \\
\hline Collaboration avec le personnel infirmier & 5.37 & 5.23 & 4.93 & 5.03 \\
\hline $\begin{array}{l}\text { Collaboration avec des services paramédicaux } \\
\text { (p.ex. physiothérapie, ergothérapie, services sociaux) }\end{array}$ & 5.50 & 5.02 & 4.65 & 4.79 \\
\hline $\begin{array}{l}\text { Collaboration avec les médecins / } \\
\text { médecins de premier recours chargés du suivi }\end{array}$ & 4.97 & 4.88 & 4.53 & 4.65 \\
\hline Evaluation réaliste de ses propres capacités & 4.80 & 4.71 & 4.50 & 4.57 \\
\hline Activité indépendante & 5.10 & 5.05 & 4.93 & 4.97 \\
\hline Principes éthiques de la médecine & 4.82 & 4.82 & 4.44 & 4.56 \\
\hline
\end{tabular}

Remarque: les valeurs allaient de 1 «très petite» (très petite contribution) à 6 «très grande». Seules ont été prises en compte les réponses de médecinsassistants exerçant leur activité dans l'établissement de formation postgraduée depuis deux mois ou plus. Pour les aspects ne concernant pas la discipline exercée, la possibilité de réponse était «non applicable». Ces valeurs n’ont pas été prises en compte dans le calcul des valeurs moyennes. 
Cohortes ayant passé les examens de fin d'études et titres de spécialiste visés par les assistants concernés

Le tableau 5 montre les titres de spécialiste visés pour les cohortes ayant passé les examens de fin d'études de 1999 à 2005. On constate des tendances semblables à celles des enquêtes précédentes [1-3]. Une partie des cohortes des années 2000, 1999 et antérieures ont déjà obtenu leur titre de spécialiste et ne figurent pour cette raison plus dans le présent sondage. A l'instar des années passées, on observe un intérêt moindre pour la psychiatrie et la psychothérapie dans la première année suivant la fin des études. Le nombre relatif des médecins-assistants visant ce titre est nettement plus petit dans la cohorte 2005 que dans les cohortes antérieures. Chez les médecins-assistants en chirurgie, on constate la tendance inverse: leur intérêt diminue au fil du temps. Parmi les médecins-assistants ayant obtenu leur diplôme de fin d'études en 2005, env. $10 \%$ briguent le titre de spécialiste en chirurgie. Ce taux s'abaisse à $5 \%$ chez ceux qui ont terminé leurs études depuis un certain temps. Les cohortes plus anciennes visent avant tout les titres de médecine interne et de psychiatrie ou les titres des 35 autres disciplines. Les titres de spécialiste en anesthésiologie, chirurgie orthopédique et médecine générale sont brigués dans toutes les cohortes à peu près selon la même fréquence. Aucun changement de direction n'a pu être constaté ici. Trois ans après l'obtention du diplôme de fin d'études, 92\% des médecins-assistants avaient déjà choisi le titre de spécialiste qu'ils voulaient obtenir.

Tableau 3

Valeurs moyennes de l'appréciation globale formulée dans les sept disciplines comptant le plus de médecinsassistants, réparties en fonction de la taille de l'établissement (MA = médecin-assistant).

\begin{tabular}{|c|c|c|c|c|c|c|c|c|}
\hline & \multicolumn{2}{|c|}{1 à $3 M A$} & \multicolumn{2}{|c|}{4 à $10 \mathrm{MA}$} & \multicolumn{2}{|c|}{$11 \mathrm{MA}$ et plus } & \multicolumn{2}{|l|}{ Total } \\
\hline & $\mathrm{N}$ & M & $\mathrm{N}$ & M & $\mathrm{N}$ & M & $\mathrm{N}$ & M \\
\hline Médecine interne & 69 & 4.96 & 347 & 5.16 & 869 & 4.77 & 1285 & 4.89 \\
\hline Anesthésiologie & 21 & 5.27 & 94 & 5.02 & 150 & 4.58 & 265 & 4.79 \\
\hline Chirurgie & 22 & 4.53 & 242 & 4.56 & 397 & 4.57 & 661 & 4.56 \\
\hline Gynécologie & 29 & 5.00 & 196 & 4.80 & 89 & 4.61 & 314 & 4.76 \\
\hline Pédiatrie & 11 & 5.18 & 93 & 4.70 & 164 & 4.74 & 268 & 4.74 \\
\hline Psychiatrie & 41 & 5.02 & 133 & 4.97 & 507 & 4.77 & 681 & 4.82 \\
\hline Chirurgie orthopédique & 30 & 4.80 & 62 & 5.10 & 106 & 4.75 & 198 & 4.87 \\
\hline
\end{tabular}

Remarque: seules ont été prises en compte les réponses de médecins-assistants exerçant leur activité dans l'établissement de formation postgraduée depuis deux mois ou plus.

Tableau 4

Valeurs moyennes de l'appréciation globale formulée dans les sept disciplines comptant le plus de médecins-assistants, réparties en fonction du type de la formation accomplie (année de formation spécifique ou année à option).

\begin{tabular}{|c|c|c|c|c|c|c|c|c|}
\hline & \multicolumn{2}{|c|}{$\begin{array}{l}\text { Année de forma- } \\
\text { tion spécifique }\end{array}$} & \multicolumn{2}{|c|}{ Année à option } & \multicolumn{2}{|c|}{$\begin{array}{l}\text { Spécialisation } \\
\text { pas encore choisie }\end{array}$} & \multicolumn{2}{|l|}{ Total } \\
\hline & $\mathrm{N}$ & $M$ & $\mathrm{~N}$ & M & $\mathrm{N}$ & $M$ & $\mathrm{~N}$ & M \\
\hline Médecine interne & 901 & 4.90 & 201 & 4.82 & 158 & 4.86 & 1260 & 4.88 \\
\hline Anesthésiologie & 231 & 4.80 & 19 & 4.67 & 14 & 4.88 & 264 & 4.79 \\
\hline Chirurgie & 386 & 4.57 & 195 & 4.60 & 68 & 4.48 & 649 & 4.57 \\
\hline Gynécologie & 276 & 4.82 & 22 & 4.53 & 11 & 4.24 & 309 & 4.78 \\
\hline Pédiatrie & 239 & 4.77 & 16 & 4.35 & 9 & 4.48 & 264 & 4.74 \\
\hline Psychiatrie & 580 & 4.84 & 65 & 4.71 & 27 & 4.69 & 672 & 4.82 \\
\hline Chirurgie orthopédique & 172 & 4.85 & 18 & 4.93 & 3 & 4.78 & 193 & 4.85 \\
\hline
\end{tabular}

Remarque: seules ont été prises en compte les réponses de médecins-assistants exerçant leur activité dans l'établissement de formation postgraduée depuis deux mois ou plus. 
Situation professionnelle: nombre d'heures consacrées par semaine à la formation postgraduée et à l'activité professionnelle. Influence de la grandeur de l'établissement et évolution de l'enquête au fil des ans

Les médecins-assistants ont été interrogés sur le nombre d'heures de formation théorique (cours académiques, cours opératoires, autres cours, séminaires) qu'ils suivaient par semaine en moyenne. Les cas extrêmes indiquant 11 heures ou plus ont été classés sous absents. En plus du temps de formation postgraduée théorique, les médecins-assistants ont indiqué leur temps de travail moyen hebdomadaire. Pour que les chiffres puissent être comparés, on a pris en considération uniquement les données des médecinsassistants exerçant leur activité à plein temps. Les cas marginaux supérieurs ( $>80 \mathrm{~h} /$ semaine) et inférieurs ( $<44 \mathrm{~h}$ /semaine) ont été éliminés. Les valeurs moyennes des sept disciplines comptant le plus de médecins-assistants figurent dans les tableaux 6 à 8 .

Le tableau 6 présente le nombre d'heures de formation postgraduée théorique suivies en moyenne par semaine dans les disciplines comptant le plus de médecins-assistants. Dans le listage du nombre d'heures de formation postgraduée et d'activité professionnelle, on a tenu uniquement compte des réponses des médecinsassistants en activité depuis deux mois au moins (au moment de l'enquête) dans l'établissement concerné et qui occupaient un poste à plein temps. Les chiffres de l'enquête 2006 sont comparables aux valeurs des années précédentes [2, 3]. Le nombre d'heures de formation postgraduée suivies est resté stable au cours des ans ou a même augmenté un peu. Aucun changement n'a été constaté en comparaison avec les enquêtes 2003 et 2004. Par rapport à 2003, on observe une légère augmentation de la fréquentation uniquement en chirurgie orthopédique.

La grandeur de l'établissement joue un rôle concernant le nombre d'heures de formation théorique suivies. Les médecins-assistants qui travaillent dans des établissements comptant de nombreux postes d'assistants, consacrent plus d'heures à la formation théorique que les médecins-assistants travaillant dans des établissements plus petits. On observe cette différence dans toutes les disciplines, à l'exception de la psychiatrie.

Les valeurs du temps de travail moyen hebdomadaire des médecins-assistants dans les sept disciplines comptant le plus de médecins-assistants figurent dans le tableau 7. Il apparaît qu'en 2006, le nombre d'heures de travail a été inférieur à celui de 2003 dans les sept disciplines. Les différences entre les disciplines n'ont guère changé. Les valeurs moyennes les plus élevées du temps de travail hebdomadaire constatées pour la chirurgie orthopédique et pour la chirurgie [1] l'ont également été en 2006.

Tableau 5

Cohortes ayant passé les examens de fin d’études de 1999 à 2005, pour les huit titres de spécialistes les plus souvent brigués.

\begin{tabular}{|c|c|c|c|c|c|c|c|c|c|c|c|c|c|c|}
\hline & \multicolumn{14}{|c|}{ Cohortes selon l'année des examens de fin d'études } \\
\hline & \multicolumn{2}{|c|}{1999} & \multicolumn{2}{|l|}{2000} & \multicolumn{2}{|l|}{2001} & \multicolumn{2}{|l|}{2002} & \multicolumn{2}{|c|}{2003} & \multicolumn{2}{|l|}{2004} & \multicolumn{2}{|l|}{2005} \\
\hline & $\mathrm{N}$ & pour cent & $\mathrm{N}$ & pour cent & $\mathrm{N}$ & pour cent & $\mathrm{N}$ & pour cent & $\mathrm{N}$ & pour cent & $\mathrm{N}$ & pour cent & $\mathrm{N}$ & pour cent \\
\hline Médecine interne & 57 & $13 \%$ & 88 & $17 \%$ & 140 & $21 \%$ & 142 & $21 \%$ & 122 & $19 \%$ & 110 & $17 \%$ & 85 & $14 \%$ \\
\hline Anesthésiologie & 37 & $8 \%$ & 34 & $7 \%$ & 44 & $7 \%$ & 40 & $6 \%$ & 36 & $6 \%$ & 44 & $7 \%$ & 30 & $5 \%$ \\
\hline Chirurgie & 16 & $4 \%$ & 25 & $5 \%$ & 44 & $7 \%$ & 44 & $6 \%$ & 53 & $8 \%$ & 50 & $8 \%$ & 60 & $10 \%$ \\
\hline Gynécologie & 35 & $8 \%$ & 29 & $6 \%$ & 30 & $4 \%$ & 43 & $6 \%$ & 36 & $6 \%$ & 49 & $7 \%$ & 38 & $6 \%$ \\
\hline Pédiatrie & 31 & $7 \%$ & 31 & $6 \%$ & 49 & $7 \%$ & 50 & $7 \%$ & 56 & $9 \%$ & 55 & $8 \%$ & 36 & $6 \%$ \\
\hline Psychiatrie & 56 & $12 \%$ & 58 & $11 \%$ & 65 & $10 \%$ & 60 & $9 \%$ & 45 & $7 \%$ & 51 & $8 \%$ & 38 & $6 \%$ \\
\hline Chirurgie orthopédique & 24 & $5 \%$ & 23 & $4 \%$ & 38 & $6 \%$ & 34 & $5 \%$ & 29 & $5 \%$ & 49 & $7 \%$ & 32 & $5 \%$ \\
\hline Médecine générale & 28 & $6 \%$ & 43 & $8 \%$ & 51 & $8 \%$ & 47 & $7 \%$ & 45 & $7 \%$ & 39 & $6 \%$ & 45 & $7 \%$ \\
\hline $\begin{array}{l}\text { Autres titres } \\
\text { de spécialiste }\end{array}$ & 161 & $36 \%$ & 172 & $33 \%$ & 196 & $29 \%$ & 191 & $28 \%$ & 156 & $25 \%$ & 129 & $19 \%$ & 130 & $21 \%$ \\
\hline $\begin{array}{l}\text { Spécialisation } \\
\text { pas encore choisie }\end{array}$ & 5 & $1 \%$ & 11 & $2 \%$ & 17 & $3 \%$ & 36 & $5 \%$ & 52 & $8 \%$ & 89 & $13 \%$ & 112 & $18 \%$ \\
\hline Total & 450 & $100 \%$ & 514 & $100 \%$ & 674 & $100 \%$ & 687 & $100 \%$ & 630 & $100 \%$ & 665 & $100 \%$ & 606 & $100 \%$ \\
\hline
\end{tabular}

Remarque: sont indiqués le nombre de mentions par année et discipline, ainsi que les pourcentages correspondants. La question était «En quelle année avezvous passé vos examens de fin d'études?» 
Dans quelques disciplines, le nombre d'heures accomplies dépend de la grandeur de l'établissement (tab. 8). Dans les établissements de formation postgraduée en anesthésiologie, chirurgie et pédiatrie, le temps de travail des médecins-assistants varie selon le nombre de médecins-assistants engagés. Néanmoins, il n'a pas été possible de déterminer une tendance générale permettant de savoir si les médecins-assistants travaillent davantage dans les petits établissements ou dans les grands.

\section{Part des médecins-assistants venant de l'étranger}

Le fait que la part de médecins-assistants possédant un diplôme officiel étranger a tendance à augmenter est également confirmé en 2006. Le tableau 9 montre qu'en 2006, près de 40\% des médecins-assistants avaient passé leur examen de fin d'études à l'étranger.

Entre les trois régions linguistiques, des différences très nettes apparaissent en ce qui concerne l'origine des médecins-assistants

Tableau 6

Valeurs moyennes du nombre d'heures de formation postgraduée théorique suivies en moyenne par semaine dans les sept disciplines comptant le plus de médecins-assistants, pour les années 2005-2006 (MA = médecin-assistant).

\begin{tabular}{|c|c|c|c|c|c|}
\hline & & \multicolumn{4}{|c|}{ Année de recensement } \\
\hline & & \multicolumn{2}{|l|}{2005} & \multicolumn{2}{|l|}{2006} \\
\hline & & $\mathrm{N}$ & M & $\mathrm{N}$ & M \\
\hline \multirow[t]{4}{*}{ Médecine interne } & 1 à $3 \mathrm{MA}$ & 77 & 1.99 & 69 & 1.82 \\
\hline & 4 à $10 \mathrm{MA}$ & 322 & 2.05 & 347 & 2.00 \\
\hline & $11 \mathrm{MA}$ et plus & 892 & 2.96 & 869 & 3.04 \\
\hline & Total & 1291 & 2.68 & 1285 & 2.70 \\
\hline \multirow[t]{4}{*}{ Anesthésiologie } & 1 à $3 \mathrm{MA}$ & 23 & 1.70 & 21 & 1.23 \\
\hline & 4 à $10 \mathrm{MA}$ & 92 & 1.51 & 94 & 1.64 \\
\hline & $11 \mathrm{MA}$ et plus & 131 & 1.98 & 150 & 2.03 \\
\hline & Total & 246 & 1.78 & 265 & 1.83 \\
\hline \multirow[t]{4}{*}{ Chirurgie } & 1 à $3 \mathrm{MA}$ & 26 & 1.36 & 22 & 1.30 \\
\hline & 4 à $10 \mathrm{MA}$ & 258 & 1.65 & 242 & 1.86 \\
\hline & $11 \mathrm{MA}$ et plus & 378 & 1.98 & 397 & 2.07 \\
\hline & Total & 662 & 1.83 & 661 & 1.97 \\
\hline \multirow[t]{4}{*}{ Gynécologie } & 1 à $3 \mathrm{MA}$ & 28 & 1.62 & 29 & 2.13 \\
\hline & 4 à $10 \mathrm{MA}$ & 201 & 1.51 & 196 & 1.64 \\
\hline & $11 \mathrm{MA}$ et plus & 80 & 2.41 & 89 & 2.16 \\
\hline & Total & 309 & 1.76 & 314 & 1.83 \\
\hline \multirow[t]{4}{*}{ Pédiatrie } & 1 à $3 \mathrm{MA}$ & 9 & 0.86 & 11 & 1.44 \\
\hline & 4 à $10 \mathrm{MA}$ & 70 & 2.08 & 93 & 1.91 \\
\hline & $11 \mathrm{MA}$ et plus & 164 & 2.82 & 164 & 2.84 \\
\hline & Total & 243 & 2.55 & 268 & 2.48 \\
\hline \multirow[t]{4}{*}{ Psychiatrie } & 1 à $3 \mathrm{MA}$ & 25 & 3.93 & 41 & 4.29 \\
\hline & 4 à $10 \mathrm{MA}$ & 106 & 3.96 & 133 & 3.93 \\
\hline & $11 \mathrm{MA}$ et plus & 364 & 4.34 & 507 & 4.42 \\
\hline & Total & 495 & 4.24 & 681 & 4.32 \\
\hline \multirow[t]{4}{*}{ Chirurgie orthopédique } & 1 à $3 \mathrm{MA}$ & 22 & 1.79 & 30 & 1.97 \\
\hline & 4 à $10 \mathrm{MA}$ & 82 & 2.05 & 62 & 2.12 \\
\hline & $11 \mathrm{MA}$ et plus & 116 & 3.30 & 106 & 3.31 \\
\hline & Total & 220 & 2.69 & 198 & 2.73 \\
\hline \multicolumn{6}{|c|}{$\begin{array}{l}\text { Remarque: seules ont été prises en compte les réponses de médecins-assistants exerçant leur activité dans } \\
\text { l'établissement de formation postgraduée depuis deux mois ou plus (uniquement emplois à plein temps). }\end{array}$} \\
\hline \multicolumn{6}{|c|}{ Les cas marginaux ont été considérés comme absents. Données en h par semaine. } \\
\hline \multicolumn{6}{|c|}{$\begin{array}{l}\text { La question était: «Combien d'heures de formation postgraduée théorique (cours, cours opératoires, autres cours } \\
\text { séminaires) avez-vous suivies en moyenne par semaine? [env. } \quad \text { h/sem.].» }\end{array}$} \\
\hline
\end{tabular}


Tableau 7

Valeurs moyennes concernant le temps de travail moyen hebdomadaire des médecins-assistants pour les sept disciplines comptant le plus de médecins-assistants, années 2003 à 2006.

\begin{tabular}{|c|c|c|c|c|c|c|c|c|}
\hline & \multicolumn{8}{|c|}{ Année de recensement } \\
\hline & \multicolumn{2}{|l|}{2003} & \multicolumn{2}{|l|}{2004} & \multicolumn{2}{|l|}{2005} & \multicolumn{2}{|l|}{2006} \\
\hline & $\mathrm{N}$ & M & $\mathrm{N}$ & M & $\mathrm{N}$ & M & $\mathrm{N}$ & M \\
\hline Médecine interne & 1156 & 57.04 & 1170 & 56.07 & 1291 & 54.48 & 1239 & 55.16 \\
\hline Anesthésiologie & 239 & 54.41 & 241 & 53.80 & 246 & 52.12 & 256 & 52.10 \\
\hline Chirurgie & 629 & 59.85 & 638 & 58.87 & 662 & 56.00 & 656 & 56.09 \\
\hline Gynécologie & 302 & 57.84 & 291 & 56.87 & 309 & 53.60 & 289 & 55.30 \\
\hline Pédiatrie & 233 & 56.85 & 240 & 54.80 & 243 & 53.82 & 244 & 53.37 \\
\hline Psychiatrie & 503 & 52.78 & 494 & 51.85 & 495 & 51.15 & 502 & 51.30 \\
\hline Chirurgie orthopédique & 186 & 61.19 & 184 & 57.70 & 221 & 56.73 & 197 & 56.47 \\
\hline Autres disciplines & 1327 & 55.73 & 1309 & 54.73 & 1458 & 53.12 & 1444 & 53.46 \\
\hline Total & 4575 & 56.67 & 4567 & 55.56 & 4925 & 53.85 & 4827 & 54.21 \\
\hline
\end{tabular}

Remarque: seules ont été prises en compte les réponses de médecins-assistants exerçant leur activité dans l'établissement de formation postgraduée depuis deux mois ou plus (uniquement emplois à plein temps). Les cas marginaux ont été considérés comme absents. Données en h par semaine.

La question était: «Combien d'heures passez-vous en moyenne par semaine sur votre lieu de travail / à l'hôpital (travail et formation postgraduée)? [env. h/sem.].»

\section{Tableau 8}

Valeurs moyennes concernant le temps de travail moyen hebdomadaire des médecins-assistants pour les sept disciplines comptant le plus de médecins-assistants, année 2006 avec répartition en fonction de la taille de l'établissement (MA = médecin-assistant).

\begin{tabular}{|c|c|c|c|c|c|c|}
\hline & \multicolumn{2}{|c|}{1 à $3 M A$} & \multicolumn{2}{|c|}{4 à $10 \mathrm{MA}$} & \multicolumn{2}{|c|}{11 MA et plus } \\
\hline & $\mathrm{N}$ & M & N & M & N & M \\
\hline Médecine interne & 66 & 56.06 & 337 & 54.56 & 836 & 55.33 \\
\hline Anesthésiologie & 20 & 49.81 & 93 & 52.94 & 143 & 51.85 \\
\hline Chirurgie & 22 & 56.50 & 240 & 54.85 & 394 & 56.82 \\
\hline Gynécologie & 27 & 53.60 & 184 & 55.82 & 78 & 54.57 \\
\hline Pédiatrie & 9 & 51.50 & 82 & 54.95 & 153 & 52.63 \\
\hline Psychiatrie & 31 & 51.94 & 94 & 52.11 & 377 & 51.05 \\
\hline Chirurgie orthopédique & 30 & 57.28 & 62 & 54.64 & 105 & 57.32 \\
\hline Total & 205 & 54.67 & 1092 & 54.53 & 2086 & 54.51 \\
\hline
\end{tabular}

Remarque: seules ont été prises en compte les réponses de médecins-assistants exerçant leur activité dans l'établissement de formation postgraduée depuis deux mois ou plus (uniquement emplois à plein temps). Les cas marginaux ont été considérés comme absents. Données en h par semaine.

La question était: «Combien d’heures passez-vous en moyenne par semaine sur votre lieu de travail / à I'hôpital (travail et formation postgraduée)? [env. h/sem.].»

Tableau 9

Lieu des examens de fin d'études des médecins-assistants, années 2003 à 2006.

\begin{tabular}{|c|c|c|c|c|c|c|c|c|}
\hline & \multicolumn{8}{|c|}{ Année de recensement } \\
\hline & 2003 & & 2004 & & 2005 & & 2006 & \\
\hline & $\mathrm{N}$ & pour cent & $\mathrm{N}$ & pour cent & $\mathrm{N}$ & pour cent & $\mathrm{N}$ & pour cent \\
\hline Suisse & 3561 & $67,8 \%$ & 3436 & $64,7 \%$ & 3555 & $62,7 \%$ & 3397 & $60,8 \%$ \\
\hline Etranger & 1692 & $32,2 \%$ & 1876 & $35,3 \%$ & 2116 & $37,3 \%$ & 2190 & $39,2 \%$ \\
\hline
\end{tabular}

Remarque: sont indiqués les chiffres absolus et les pourcentages par colonne. La question était: «Dans quel pays avez-vous passé vos examens de fin d'études?» 
(tab. 10). La part relative de médecins-assistants qui ont obtenu leur diplôme de fin d'études en Suisse est la plus grande en Suisse romande. Au Tessin, une grande partie $(43,3 \%)$ des médecinsassistants ont obtenu leur diplôme en Italie. En Suisse alémanique, env. 36\% d'entre eux possèdent un diplôme allemand.

Des différences importantes sont également observées entre disciplines. La part des médecins-assistants ayant achevé leurs études à l'étranger est particulièrement élevée en chirurgie orthopédique (62\%) ainsi qu'en psychiatrie et psychothérapie (60\%).

\section{Discussion}

Les tendances observées ces dernières années ont été généralement confirmées par l'enquête 2006. A l'instar des années précédentes, notre dernière enquête a montré que, dans l'ensemble, la formation postgraduée fonctionne bien en Suisse. Quelques établissements affichent des valeurs faibles qui sont préoccupantes. Mais, globalement, les établissements sont évalués de façon positive, ce qui est réjouissant.

Le niveau élevé de la formation postgraduée en Suisse se révèle clairement lorsque l'on procède à une comparaison avec l'évaluation de la formation postgraduée en Allemagne. Une enquête effectuée en 2006 dans les cliniques HELIOS S.àr.l., soit l'une des chaînes de cliniques occupant une place de leader sur le marché en Allemagne, a montré que la formation postgraduée était nettement mieux évaluée en Suisse qu'en Allemagne (cliniques HELIOS) [4] dans les huit dimensions mesurées (évaluation globale, etc.). Ce résultat pourrait expliquer le nombre élevé de médecins-assistants allemands travail- lant en Suisse. La qualité de la formation dispensée, un revenu élevé et une atmosphère de travail agréable font partie des facteurs rendant le travail en Suisse attrayant.

Depuis 2003, on constate une diminution du temps de travail moyen hebdomadaire des médecins-assistants d'env. 2,5 heures. Cette diminution est clairement due à l'introduction de la loi sur le travail [1]. Mais selon l'évaluation subjective des médecins-assistants, la prescription légale de la semaine de 50 heures n'a pu, cette année aussi, être respectée dans aucune des sept disciplines comptant le plus de médecins-assistants. La réduction du temps de travail hebdomadaire semble stagner.

La réduction du temps de travail ainsi que les nouvelles prescriptions concernant les tours de service (travail par roulement) ont entraîné, entre autres, une augmentation du nombre de places de formation postgraduée de $8 \%$ entre 2003 et 2006. Durant cette même période, la part de médecins-assistants provenant de l'étranger a augmenté de 7\%. En parallèle, le nombre de diplômes de fin d'études suisses est resté relativement constant, ce qui révèle un déséquilibre entre le besoin nouvellement créé de médecinsassistants et le nombre de diplômes de médecin octroyés en Suisse.

L'enseignement des connaissances professionnelles spécifiques obtient des valeurs très élevées dans tout l'échantillon des personnes sondées. La formation donnée dans les domaines «accompagnement de mourants» et «évaluation de la capacité de travail du patient» est plutôt mal notée dans toutes les disciplines. Les compétences ne sont pas toutes d'importance égale pour les disciplines, ce qui explique la valeur basse

\begin{tabular}{|c|c|c|c|c|c|c|c|c|}
\hline \multicolumn{9}{|l|}{ Tableau 10} \\
\hline \multicolumn{9}{|c|}{$\begin{array}{l}\text { Pays dans lequel les examens de fin d'études ont été passés; répartition selon la région linguistique où se trouve } \\
\text { l'établissement de formation des médecins-assistants concernés. }\end{array}$} \\
\hline & \multicolumn{8}{|c|}{ Région } \\
\hline & \multicolumn{2}{|c|}{ Suisse alémanique } & \multicolumn{2}{|c|}{ Suisse romande } & \multicolumn{2}{|c|}{ Tessin } & \multicolumn{2}{|l|}{ Total } \\
\hline & $\mathrm{N}$ & pour cent & $\mathrm{N}$ & pour cent & $\mathrm{N}$ & pour cent & $\mathrm{N}$ & pour cent \\
\hline Suisse & 2263 & $57,1 \%$ & 1052 & $74,1 \%$ & 82 & $40,8 \%$ & 3397 & $60,8 \%$ \\
\hline Allemagne & 1440 & $36,3 \%$ & 88 & $6,2 \%$ & 13 & $6,5 \%$ & 1541 & $27,6 \%$ \\
\hline France & 1 & $<0,1 \%$ & 26 & $1,8 \%$ & 0 & $0,0 \%$ & 27 & $0,5 \%$ \\
\hline Italie & 9 & $0,2 \%$ & 30 & $2,1 \%$ & 87 & $43,3 \%$ & 126 & $2,3 \%$ \\
\hline Autriche & 114 & $2,9 \%$ & 6 & $0,4 \%$ & 0 & $0,0 \%$ & 120 & $2,1 \%$ \\
\hline Reste de l'UE & 53 & $1,3 \%$ & 77 & $5,4 \%$ & 4 & $2,0 \%$ & 134 & $2,4 \%$ \\
\hline Autres pays & 86 & $2,2 \%$ & 141 & $9,9 \%$ & 15 & $7,5 \%$ & 242 & $4,3 \%$ \\
\hline
\end{tabular}

Remarque: sont indiqués les chiffres absolus et les pourcentages par colonne. La question était: «Dans quel pays avez-vous passé vos examens de fin d'études?» 
accordée à l'aptitude à évaluer la capacité de travail du patient en pédiatrie et les valeurs tout aussi basses enregistrées pour le domaine de l'«accompagnement de mourants» dans les établissements des branches opératives, de la pédiatrie ainsi que de la psychiatrie et psychothérapie. Dans ces disciplines, des décès surviennent rarement lors d'une hospitalisation. Dans les établissements de formation postgraduée en chirurgie et chirurgie orthopédique, une plus grande importance devrait être accordée à l'enseignement portant sur l'utilisation correcte des médicaments, et ce dans l'intérêt de la sécurité des patients.

Les cohortes des examens de fin d'études permettent d'observer des schémas clairs dans le choix des disciplines. Dans les cohortes plus âgées, la part des psychiatres est supérieure à celle observée dans les cohortes plus jeunes. L'inverse se produit chez les chirurgiens. Le fait que les médecins-assistants commencent par viser le titre de spécialiste en chirurgie pour choisir ensuite un autre titre s'explique de différentes manières. Au début de leur formation postgraduée, ils sont sans doute enthousiasmés par la discipline puis ils remarquent au fil des ans qu'ils n'ont pas l'habileté manuelle nécessaire ou qu'ils s'étaient fait une image différente de la chirurgie. La question se pose ici de savoir comment on peut améliorer la sélection et déterminer plus tôt si un médecinassistant convient pour la chirurgie. Les différences entre les cohortes ayant choisi le titre de spécialiste en psychiatrie et psychothérapie peuvent être interprétées de la façon suivante: les psychiatres ont probablement besoin de plus de temps que les médecins-assistants des autres disciplines pour obtenir leur titre de spécialiste. Cette interprétation s'appuie sur le fait que les médecins-assistants en psychiatrie (surtout dans les cohortes plus âgées) sont plus nombreux à travailler à temps partiel que dans les autres disciplines. Il est aussi possible que les médecins-assistants n'optent pour la psychiatrie que tardivement.

Le nombre d'heures de formation théorique suivies par les médecins-assistants est plus élevé dans les grands établissements que dans les petits. Une des raisons pourrait en être que les grands établissements offrent plus de formation postgraduée théorique et que les médecins-assistants y sont moins soumis à des tours de service (travail par roulement).

\section{Regard vers I'avenir}

Dans le cadre de l'enquête statistique pour 2007, il faudra demander aux responsables d'établissements de formation postgraduée quelle est, selon eux, la contribution fournie par leur établissement au développement des compétences dans la discipline concernée. Leur réponse permettra d'effectuer une comparaison entre élèves et enseignants. Le retour d'information qui sera communiqué aux responsables 2007 devra ensuite, si possible, être adapté encore mieux aux diverses disciplines en ce qui concerne la transmission des compétences.

L'étendue des interventions et examens cliniques [5] ainsi que le mode de transmission des compétences [6] dans les établissements de formation postgraduée devront, dans un avenir proche, faire l'objet de discussions approfondies au sein des disciplines. En renforçant les réseaux et la collaboration régionale et nationale entre établissements de formation postgraduée, de même qu'en appliquant des méthodes d'enseignement modernes, il sera possible d'augmenter la fréquentation des cours théoriques de formation postgraduée aussi dans les petits établissements.

\section{Références}

1 Siegrist M, Orlow P, Giger M. Principaux résultats de l'enquête effectuée en 2005 auprès des médecins-assistants: Formation postgraduée et situation professionnelle vues par les médecins-assistants. Bull Méd Suisses. 2006;87(10):379-86.

2 Siegrist M, Orlow P, Giger M. Weiterbildung aus der Sicht der Assistenzärzte: Die wichtigsten Resultate der Umfrage 2004 bei Assistenzärztinnen und -ärzten über die Weiterbildung. Schweiz Ärztezeitung. 2005;86(7):412-23.

3 Siegrist M, Gutscher H, Giger M. Was haben Betriebskultur, Motivation und Fremdjahr mit der Weiterbildung zu tun? Schweiz Ärztezeitung. 2004;85(15):783-91.

4 Fotuhi P, Siegrist M, Orlow P, Vogel S, Giger M. Beurteilung der Weiterbildung der Assistenzärzte in der Schweiz und in Deutschland (HELIOS Kliniken). Schweiz Ärztezeitung. 2006;87(48):2081-3.

5 Duffy FD, Holbboe ES. What procedures should internists do? Ann Intern Med. 2007;146:392-3.

6 Reznick RK, MacRae H. Teaching surgical skills changes in the wind. N Engl J Med. 2006;355: 2664-9. 